Original Paper http://ajol.info/index.php/ijbcs http://indexmedicus.afro.who.int

\title{
Niveau de connaissance et composition minérale de Hydrocotyle bonariensis Lam. (Araliaceae), une plante utilisée dans les ménages du District d'Abidjan (Côte d'Ivoire)
}

\author{
Ebalah Delphine MONYN ${ }^{1}$, Adama BAKAYOKO ${ }^{1,2}$, Fézan Honora TRA BI ${ }^{1}$, \\ Konan $\mathrm{YAO}^{2}$, et Mamidou Witabouna $\mathrm{KONE}^{1,2^{*}}$ \\ 1 UFR Sciences de la Nature, Université Nangui Abrogoua, BP 801 Abidjan 02, Côte d'Ivoire. \\ 2 Centre Suisse de Recherches Scientifiques en Côte d'Ivoire, BP 1303 Abidjan 01, Côte d'Ivoire. \\ *Auteur correspondant; E-mail: konewit_sn@una.edu.ci; mamidou.kone@csrs.ci, Tel: + 22503488905.
}

\section{RESUME}

Hydrocotyle bonariensis est une espèce végétale entrant dans les habitudes alimentaires et médicinales des populations en Côte d'Ivoire. Ce travail a été mené afin de déterminer le niveau de connaissance et d'utilisation de cette plante dans les ménages du district d'Abidjan ainsi que sa composition en micronutriments. Au total, 800 ménages répartis dans six communes du district ont été interviewés à partir d'un questionnaire. Le dosage des minéraux a été fait au spectrophotomètre d'adsorption atomique à flamme. Ces interviews ont inclus 597 femmes et 203 hommes. Leur niveau d'éducation varie de aucun niveau (382 personnes) à supérieur (61 personnes). Sur 513 ménages ayant une connaissance de la plante, 267 l'utilisent pour des besoins médicinaux et alimentaires. Le niveau de consommation (27\%) est influencé par les niveaux d'instruction et de vie et les groupes culturels ; les Akan du Centre en sont les plus grands consommateurs. Les organes les plus utilisés sont les feuilles qui ont une composition minérale en calcium (60\%), magnésium (35\%), fer (3\%), zinc (1\%) et en cuivre (1\%). Cette étude constitue une première étape dans la valorisation nutritionnelle de $H$. bonariensis.

(C) 2016 International Formulae Group. All rights reserved.

Mots clés: Ethnobotanique, connaissance, Hydrocotyle bonariensis, minéraux, Abidjan, Côte d'Ivoire.

\section{Knowledge level and mineral content of Hydrocotyle bonariensis Lam. (Araliaceae), a plant used by households in the District of Abidjan (Cote d'Ivoire)}

\begin{abstract}
Hydrocotyle bonariensis is a plant species entering in food and medicinal habits of people in Cote d'Ivoire. This work was conducted to determine the households' knowledge and utilization of this plant in the district of Abidjan as well as it mineral content. A total of 800 households in six settings were interviewed using a questionnaire. The determination of mineral content was carried out using an atomic adsorption spectrophotometer. The surveyed people included 597 women and 203 men. Their education level varied from no formal education level (382 people) to high school level (61 people). Of 513 households having a
\end{abstract}


knowledge about this plant, 267 used it for medicinal and food needs. The level of consumption (27\%) is influenced by education and life level and cultural groups; Akan from the Central Cote d'Ivoire were the largest consumers. The most used organs were the leaves that had mineral content of calcium (60\%), magnesium (35\%), iron (3\%), zinc (1\%) and copper (1\%). This study is a first step for the nutritional valorization of $H$. bonariensis.

(C) 2016 International Formulae Group. All rights reserved.

Keywords: Ethnobotany, Knowledge, Hydrocotyle bonariensis, Minerals, Abidjan, Cote d'Ivoire.

\section{INTRODUCTION}

La malnutrition par carences en micronutriments (vitamines et sels minéraux), encore appelée «faim invisible» ou «faim cachée» est très importante et touche près de deux milliards de personnes dans le monde (FAO, 2016). Selon cette même source, l'Organisation Mondiale de la Santé (OMS) estime que les carences en fer, en vitamine A et en zinc sont parmi les dix premières causes de décès par maladie dans les pays en développement. Elles touchent le plus souvent les personnes vulnérables tels que les enfants, les femmes enceintes et les vieillards. Ces carences en un ou plusieurs oligoéléments constituent donc un véritable problème de santé publique. Dans ces pays en développement, l'apport en certains minéraux comme le fer n'est pas souvent couvert par l'alimentation. En outre, la faible biodisponibilité des nutriments dans l'alimentation représente l'un des plus grands problèmes dans la lutte contre les carences nutritionnelles.

En Afrique, les espèces végétales sauvages jouent un rôle important dans l'alimentation des populations (Diarra et al., 2016). Les plantes vertes comestibles pourraient aider à la prise en charge des malnutritions en micronutriments d'autant plus qu'elles sont facilement accessibles aux communautés à cause de leur faible coût (Hailu et Addis, 2016). En Côte d'Ivoire (N'Guessan et al., 2009; Kouamé et al., 2015), bien que les populations aient recours à des plantes médicinales et/ou alimentaires cultivées ou spontanées, Atchibri et al. (2012) rapportent que les légumes feuilles n'occupent pas une place de choix dans le régime alimentaire. Or plusieurs auteurs ont montré que les légumes feuilles issues des plantes spontanées augmente la qualité nutritionnelle des mets en fournissant des minéraux, des fibres, des vitamines et des acides gras essentiels (Adeniyi et al., 2012 ; Adjatin et al., 2013 ; Volpe et al., 2015 ; Guarrera et Savo, 2016 ; Petropoulos et al., 2017). Ces légumes feuilles verts encore sous utilisés sont de bonnes sources de nombreux nutriments comme le fer, le calcium, l'acide ascorbique et le $\beta$-carotène. Selon Sanclemente et al. (2011), leurs richesses en oligoéléments, vitamines et phytocomposés sont une aubaine dans les pays en développement.

L'évaluation des compositions en nutriments et anti-nutriments des plantes spontanées comestibles pourrait aider à identifier des aliments riches en minéraux et acquérir une connaissance des méthodes appropriées de préparation pour améliorer la biodisponibilité de ces nutriments (Hailu et Addis, 2016). Dans ce cadre, différents travaux menés sur quelques plantes alimentaires de Côte d'Ivoire ont porté sur leurs valeurs nutritionnelles et diététiques (Soro et al., 2012 ; Atchibri et al., 2012 ; Acho et al., 2014). Cependant, leur importance et leur rôle dans l'alimentation des populations urbaines restent encore insuffisamment documentés. La valeur de nombreuses espèces pour les populations, éventuellement celles utilisées pour des applications médicales et nutritionnelles, est encore très peu étudiée. Dans cet arsenal des plantes disponibles, Hydrocotyle bonariensis, une plante de la famille des Araliaceae a attiré notre attention.

$H$. bonariensis est une plante, herbacée vivace, rampante, s'enracinant aux nœuds et se 
développant en tache dense. De son pivot principal robuste, elle se propage par des racines pivotantes adventives qui naissent à chaque nœud en contact avec le sol. Dans plusieurs pays, cette plante est utilisée dans la médecine populaire et dans l'alimentation (Andrea, 2010 ; Fiaschi, 2010). En Côte d'Ivoire, cette espèce végétale est aussi consommée dans des ménages, toutefois très peu de données sont disponibles sur ses usages et sa connaissance.

Ce travail a été entrepris afin de déterminer le niveau de connaissance de cette plante et sa composition minérale en vue d'une valorisation nutritionnelle et médicinale.

\section{MATERIEL ET METHODES}

\section{Zone d'étude}

Les enquêtes ont été menées dans le district d'Abidjan (Figure 1) qui se trouve sur le littoral Sud-Est de la Côte d'Ivoire. C'est la capitale économique du pays. Elle comptait en 2014, 4707000 habitants soit 20\% de la population totale du pays (INS, 2015). Les six communes visitées sont Port-Bouët, Abobo, Yopougon, Cocody, Bingerville et Anyama. Elles ont été choisies par rapport à leur population de niveau de vie faible à élevé. Par ailleurs, une pré-enquête a montré que plusieurs résidents utilisent Hydrocotyle bonariensis pour divers besoins.

\section{Authentification de l'espèce végétale}

Plusieurs échantillons de la plante ont été récoltés sur divers sites et authentifiés à l'Université Nangui Abrogoua, en raison de la variabilité morphologique et d'une confusion possible avec une autre espèce, Centella asiatica.

\section{Enquêtes auprès des ménages Calcul de l'échantillon}

L'unité d'échantillonnage est le menage. La taille de l'échantillon a été calculée selon la méthode décrite par Vessereau (1992).

$$
n=t^{2} \times p(1-p) / m^{2}
$$

avec $\mathrm{n}=$ taille d'échantillon requise, $\mathrm{t}=1,96$ à un niveau de confiance de $95 \%, \mathrm{~m}=$ marge d'erreur à 4,14\%. Dans ce travail, il a été considéré une valeur de $\mathrm{p}=50 \%$ afin d'obtenir un échantillon maximal (Bouvet et Grimont, 2001) ; le niveau de connaissance de Hydrocotyle bonariensis étant indéterminé. Ainsi un échantillon de 800 ménages à enquêter a été obtenu et reparti proportionnement à la taille de chacune des six communes choisies (150 à Abobo, 150 à Anyama, 150 à Yopougon, 150 à Port-Bouët, 150 à Bingerville et 50 à Cocody).

Enquêtes

Des séries d'enquêtes ethnobotanique et alimentaire ont été menées dans les six communes choisies. Ces enquêtes semi structurées ont été conduites durant la période allant de Décembre 2014 à Mars 2016 à l'aide d'une fiche d'enquête. Les ménages ont été tirés de manière aléatoire. L'enquête a consisté à interroger la maîtresse ou l'homme de la maison. Les grandes lignes de l'interview concernaient les informations sur la plante, son utilisation alimentaire, dans les soins de santé, le mode de préparation, la fréquence de consommation et sur d'autres usages de Hydrocotyle bonariensis. Les caractéristiques socio-démographiques ont été prises en compte dans l'enquête. Les langues de communication étaient le français et les langues locales comme le Dioula et le Baoulé.

\section{Détermination de la teneur en minéraux Minéralisation}

Une quantité de 0,3 g d'échantillon broyé a été pesée dans un creuset en porcelaine puis mise dans un four à moufle (PROLABO) à $650{ }^{\circ} \mathrm{C}$ pendant $5 \mathrm{~h}$. Après refroidissement, $5 \mathrm{~mL}$ d'acide nitrique (1 mol. $\mathrm{L}^{-1}$ ) ont été ajoutés à la cendre obtenue puis portés à évaporation totale sur un bain de sable. Au résidu ont été ajoutés $5 \mathrm{~mL}$ d'acide chlorhydrique $\left(0,1 \mathrm{~mol} . \mathrm{L}^{-1}\right)$, le tout a été remis au four à $400{ }^{\circ} \mathrm{C}$ pendant $30 \mathrm{~min}$. Le résidu final a été récupéré avec $10 \mathrm{~mL}$ d'acide chlorhydrique $\left(1 \mathrm{~mol} . \mathrm{L}^{-1}\right)$ puis versé dans une fiole de $50 \mathrm{~mL}$. Le creuset a été rincé deux 
fois avec $10 \mathrm{~mL}$ de l'acide chlorhydrique. La fiole a été complétée à $50 \mathrm{~mL}$ avec l'acide chlorhydrique. Dans les mêmes conditions, un essai à blanc a été réalisé (IITA, 1981 ; AOAC, 1990).

\section{Dosage}

Les minéraux dosés ont été le calcium, le cuivre, le fer, le magnésium, le phosphore et le zinc. Après la minéralisation, des gammes de concentrations de chaque minéral ont été préparées selon les méthodes de références pour la détermination quantitative des éléments chimiques dans les végétaux (IITA, 1981). Les dosages ont été réalisés à l'aide d'un spectrophotomètre d'adsorption atomique à flamme air-acétylène de type VARIAN AAS 20, de marque Australienne (AOAC, 1990 ; Kouassi et al., 2013). Les dosages ont été répétés trois fois.

\section{Traitement des données}

\section{Pourcentage de connaissance}

Le pourcentage de connaissance a été calculé par le rapport du nombre de ménages connaissant la plante $(\mathrm{N})$ et du nombre total de ménages interrogés $(\mathrm{Nt})$ selon la formule suivante $: \mathbf{N C}=\mathbf{N} / \mathbf{N t} \times \mathbf{1 0 0}$.

\section{Indice de consensus}

Afin d'apprécier les accords des informateurs sur l'utilisation médicinale de la plante contre les affections énumérées sur le plan médicinal, un facteur (degré) de consensus d'utilisation ou Informant Consensus Factor (ICF) a été calculé selon la formule suivante :

ICF $=\mathbf{N a} / \mathbf{N t}$; où $\mathrm{Na}$ est le nombre d'informateurs ayant cité une pathologie et $\mathrm{Nt}$ le nombre total d'informateurs (Ilumbe et al., 2014).

L'ICF varie entre [0-1]. Une valeur faible, proche de 0 , indique que les informateurs sont en désaccord sur les thérapies proposées. Une valeur élevée, proche de 1 , indique un consensus élevé ou total autour de l'usage de la plante.

\section{Pourcentage de consommation}

Les pourcentages de consommation dans les six communes du district d'Abidjan ont été calculés selon la méthode d'Ambé (2001). Ce pourcentage a été calculé par le rapport du nombre de ménages consommant la plante (n) et du nombre total de ménages connaissant la plante $(\mathrm{N})$ selon la formule suivante : $\mathbf{P C}=\mathbf{n} / \mathbf{N} \times \mathbf{1 0 0}$

Proportion de chaque minéral dans l'échantillon

Le pourcentage en minéral $(\mathrm{Pm})$ a été obtenu à l'aide de la formule

$\mathbf{P m}=(\mathbf{T m} / \mathbf{T}) \times \mathbf{1 0 0}$, où $\mathrm{Tm}=$ teneur $\mathrm{du}$ minéral et $\mathrm{T}=$ Teneur total pour l'ensemble des minéraux identifiés.

\section{Analyses statistiques}

La saisie des données a été réalisée avec le logiciel Epidata 3.1 et transférée sur le logiciel SPSS 20.0 pour la mise en place de la base de données. Le test de Khi-deux $\left(\chi^{2}\right)$ a été utilisé pour établir, s'il existe, une relation entre le niveau de connaissance, d'utilisation et de consommation de Hydrocotyle bonariensis et des paramètres tels que le lieu d'habitation et le groupe culturel, à l'aide du logiciel SPSS 20.0. Lorsqu'une relation existe au seuil de $0,05 \%$, le test de Khi-deux est complété par la procédure de Marascuilo (Marascuilo et Serlin, 1988) à l'aide du logiciel X1Stat version 2014. Cette procédure consiste à effectuer des tests de comparaison deux à deux pour tous les couples de proportions, ce qui permet d'identifier les proportions responsables de l'existence de liaison. La plus petite différence significative a été fixée au seuil de 0,05 (Vessereau, 1992).

\section{RESULTATS \\ Caractéristiques sociodémographiques}

$\mathrm{Au}$ total, 800 personnes dont 203 hommes et 597 femmes, ont été interviewées. Les personnes n'ayant aucun niveau sont les plus nombreuses, avec un total de 382. Les communes d'Abobo et de Bingerville en comptent le plus grand nombre, respectivement 87 et 83 personnes. Trois classes d'âge se dégagent à savoir: 18-30 ans ; 31-45 et supérieure ou égale à 46 ans. Les groupes ethniques sont marqués par la prépondérance des Akan du Centre et les Krou 
qui ont été plus nombreux à Yopougon et Port-Bouët. Quant à la situation sociale, les commerçants et les ménagères en sont les plus nombreux, avec Bingerville ayant compté plus de ménagères (Tableau 1)

\section{Niveaux de connaissance et d'utilisation de} Hydrocotyle bonariensis par commune

Sur les 800 ménages interrogés, $64 \%$ connaissent la plante et $36 \%$ non. Le test de Khi-deux montre une différence significative entre ces pourcentages. Ce résultat indique que cette plante est connue des populations du district d'Abidjan. Pour les communes d'Abobo, d'Anyama et de Cocody, le niveau de connaissance est le même mais moins élevé que celui de Yopougon, Port-Bouët et Bingerville. Il y a donc une différence significative entre ces deux groupes de communes, Bingerville a le plus haut niveau de connaissance (Figure 2).

Sur $64 \%$ de ménages connaissant la plante, $52 \%$ l'utilisent et $48 \%$ non. On constate ici que la connaissance est liée à l'utilisation car les deux groupes de communes que nous avons identifiés sont statistiquement les mêmes. A Abobo, Anyama et Cocody, la plante est faiblement utilisée par rapport à Yopougon, Port-Bouët et Bingerville. Yopougon et Port-Bouët montrent un niveau moyen et Bingerville le plus haut niveau d'utilisation (Figure 3).

Niveaux de connaissance et d'utilisation de Hydrocotyle bonariensis par groupe ethnique

Au niveau de la connaissance de cette plante, il n'y a pas de différence significative entre les différents groupes culturels. Néanmoins, les Mandé du Nord représentent la plus faible proportion (Figure 4). Pour les huit groupes culturels, les proportions sont égales $(\mathrm{F}=14,230 ; p<0,001)$. Ceci montre qu'il y a une similarité entre le niveau de connaissance de la plante quel que soit le groupe culturel auquel appartient la personne interrogée sauf pour le groupe des Mandé du Nord.
Deux classes se dégagent quant à l'utilisation de la plante (Figure 5). Ce sont la classe des groupes culturels à forte utilisation (Akan du centre et ressortissants des pays CEDEAO) et la classe des groupes à faible utilisation (Mandé du Nord, ceux du Sud, les Akan du Sud, ceux de l'Est, les Gour et les Krou).

Niveau de consommation de Hydrocotyle bonariensis par commune et par groupe ethnique

Sur l'ensemble des ménages qui utilisent cette plante, $48 \%$ en font un usage médicinal, $44 \%$ un usage alimentaire et $8 \%$ de ménages y ont recours à la fois comme aliment et comme médicament.

Au niveau des communes, Bingerville est celle qui montre le plus haut niveau de consommation, suivie d'Abobo et Cocody avec un niveau moyen de consommation. Port-Bouët, Anyama et Yopougon viennent avec une faible consommation de la plante (Figure 6).

Sur le plan culturel, les huit groupes ethniques consomment cette plante mais ce sont surtout les Akan du Centre qui la consomment davantage (Figure 7).

\section{Niveau d'instruction des ménages interrogés et consommation de Hydrocotyle bonariensis \\ Le niveau d'instruction a une influence} significative sur le niveau de consommation (relativement faible de 27\%) des feuilles de Hydrocotyle bonariensis dans les différents ménages (Figure 8). En effet, les ménages sans aucun niveau d'instruction représentent la plus forte proportion de consommateurs $(47,75 \%)$, suivis des niveaux primaire $(22,5 \%)$, secondaire $(20,12 \%)$ et supérieur $(7,625 \%)$. Quant au niveau coranique, il représente la plus faible proportion (2\%).

\section{Usages médicinaux et indice de consensus}

A l'issu de cette enquête, il ressort que Hydrocoytyle bonariensis rentre dans le traitement de nombreuses maladies parmi 
lesquelles on peut citer le diabète, l'hypertension, les maladies du système digestif, les maladies immunitaires, les maladies liées à la reproduction, etc... (Tableau 2). Les indices de consensus calculés donnent de faibles valeurs allant de 0,006 à 0,27 . Aucun indice n'a atteint une valeur moyenne de consensus $(0,5)$. Les valeurs de consensus les plus élevées ont été obtenues pour les maladies immunitaires $(\mathrm{ICF}=0,27$ ), les maladies du système digestif $(\mathrm{ICF}=0,17)$, l'hypertension $(\mathrm{ICF}=0,12)$.

\section{Usages alimentaires et indice de consensus}

Sur le plan alimentaire, trois modes de préparation sont utilisés dans les ménages

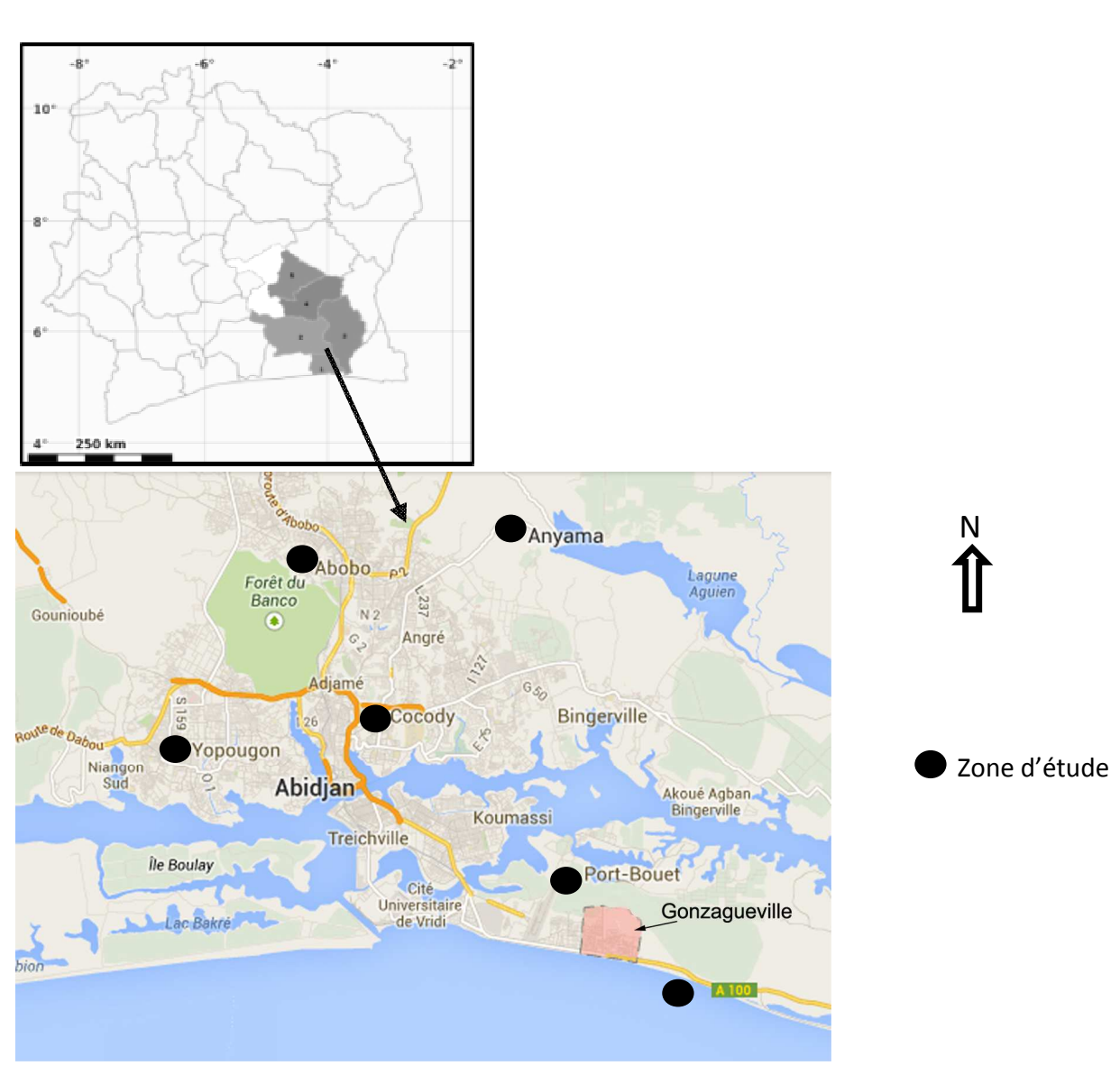

Figure 1 : Carte de localisation des sites d'étude (Source : Données cartographiques 2016).
(Tableau 3). La consommation des feuilles sous forme de crudité est la plus utilisée avec un ICF de 0.88. Cette valeur élevée indique qu'il y a un accord plus important pour cette forme de consommation que les autres.

\section{Composition en oligoéléments}

L'analyse des minéraux a permis de montrer que cette plante est riche en calcium $(60 \%)$ et en magnésium $(35 \%)$ et contient de très faibles quantités de fer $(3 \%)$, de zinc $(1 \%)$ et de cuivre $(1 \%)$ comme l'indique la Figure 9. Elle ne renferme pas de phosphore. Les teneurs observées pour chaque minéral sont consignées dans le Tableau 4. 


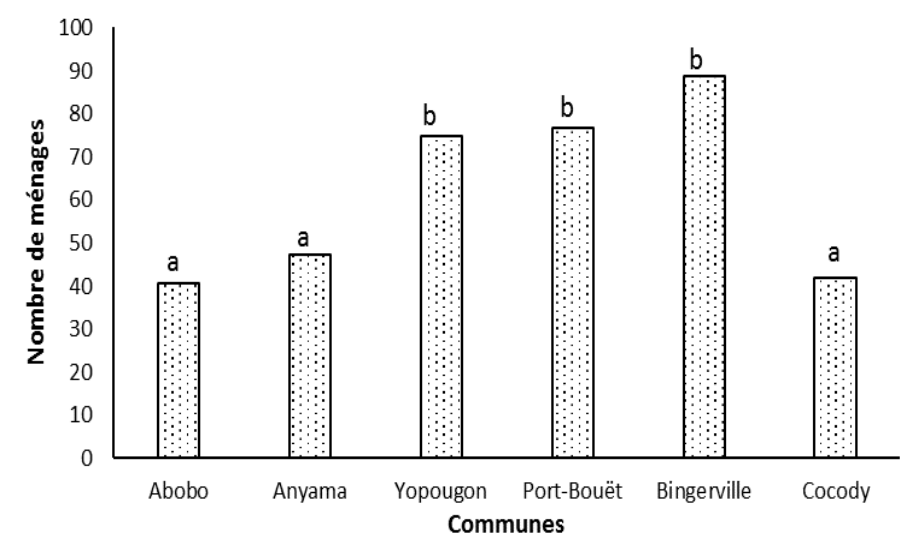

Figure 2 : Diagramme montrant le nombre de personnes connaissant Hydrocotyle bonariensis par commune.

Les bandes portant les mêmes lettres en haut ne sont pas significativement différentes $(\mathrm{p}=0,001)$.

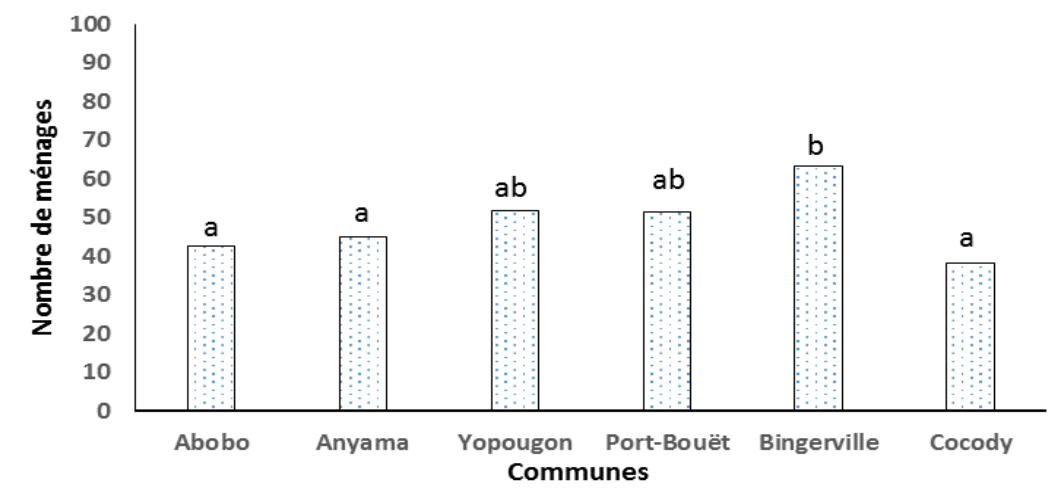

Les bandes portant les mêmes lettres en haut ne sont pas significativement différentes $(p=0,038)$.

Figure 3 : Diagramme montrant le nombre de personnes utilisant Hydrocotyle bonariensis par commune.

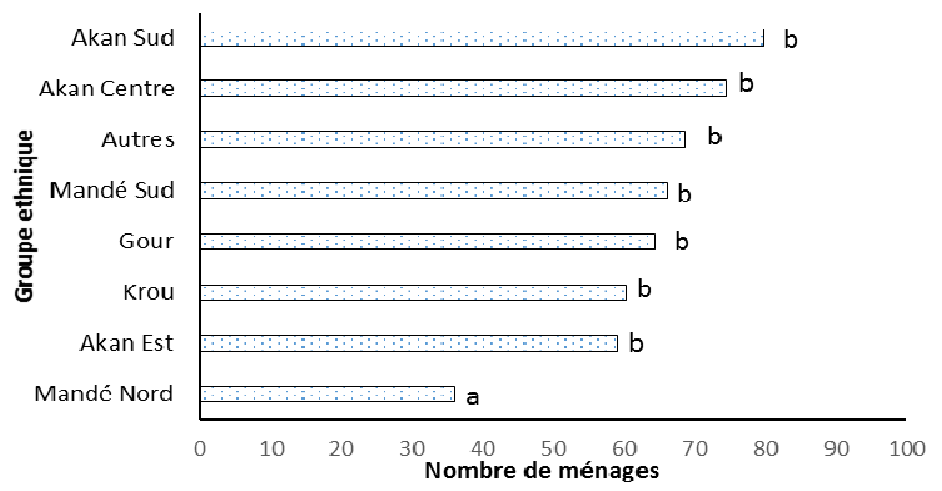

Figure 4 : Diagramme montrant le nombre de personnes connaissant Hydrocotyle bonariensis par groupe ethnique.

Les bandes portant les mêmes lettres en haut ne sont pas significativement différentes $(\mathrm{p}=0,001)$ Autres: ressortissants des pays CEDEAO. 


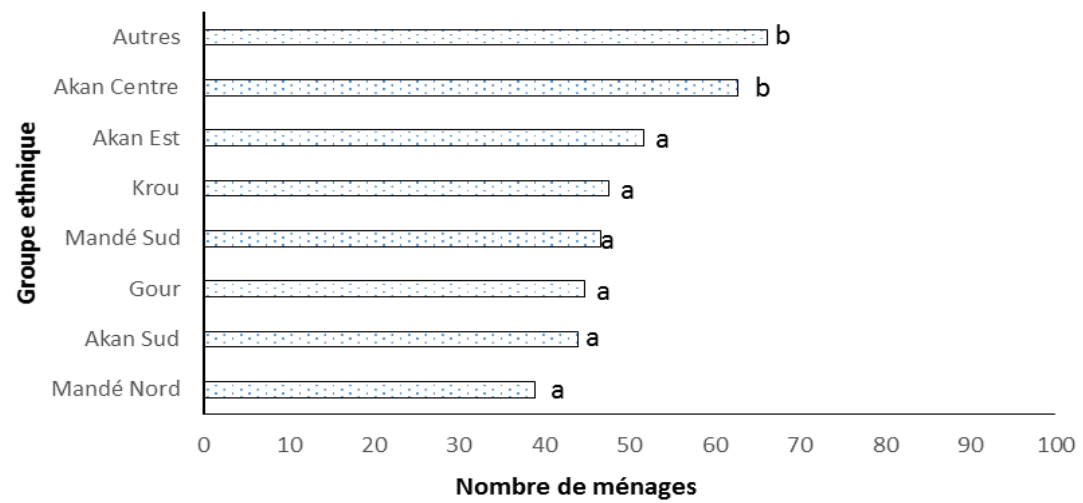

Figure 5 : Diagramme montrant le nombre de personnes utilisant Hydrocotyle bonariensis par groupe ethnique.

Les bandes portant les mêmes lettres en haut ne sont pas significativement différentes $(\mathrm{p}=0,038)$ Autres : ressortissants des pays CEDEAO.

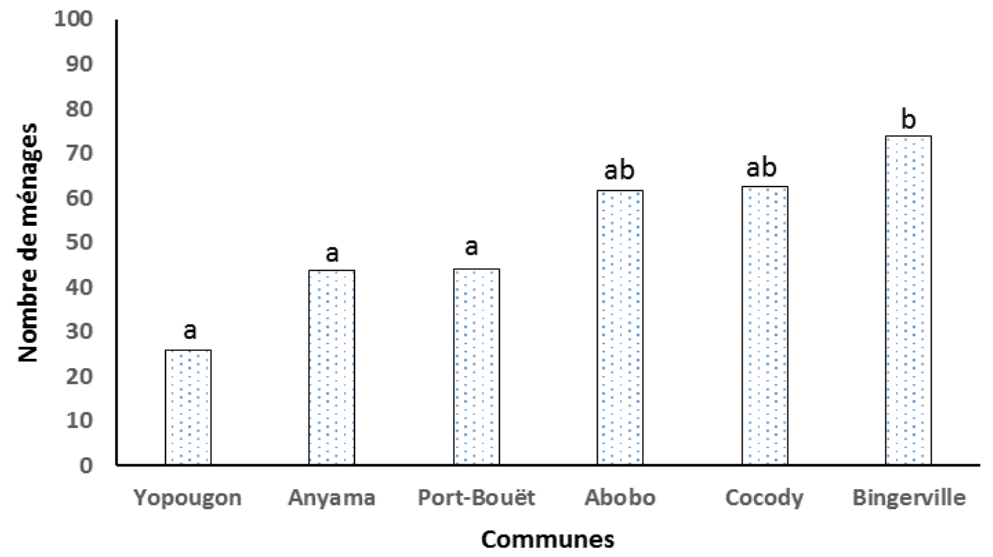

Figure 6 : Diagramme montrant le nombre de personnes consommant Hydrocotyle bonariensis par commune.

Les bandes portant les mêmes lettres en haut ne sont pas significativement différentes.

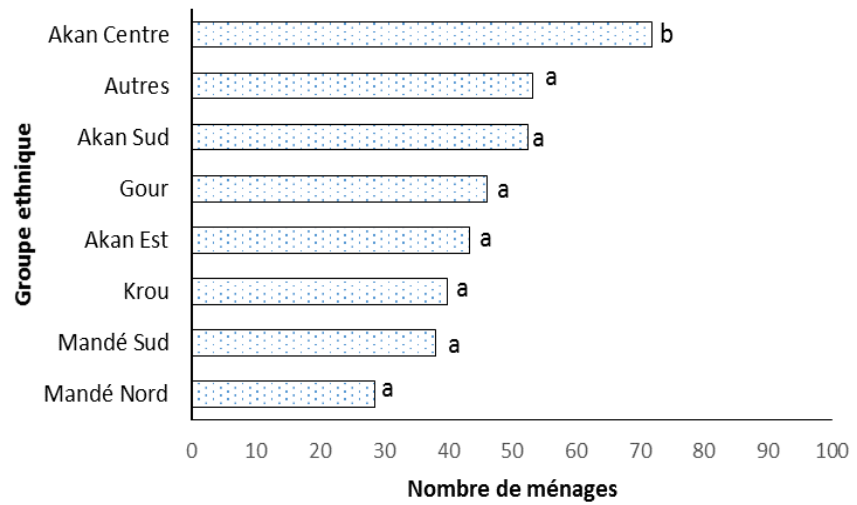

Figure 7 : Diagramme montrant le nombre de personnes consommant Hydrocotyle bonariensis par groupe ethnique. Les bandes portant les mêmes lettres en haut ne sont pas significativement différentes $(\mathrm{p}=0,038)$ Autres : ressortissants des pays CEDEAO. 


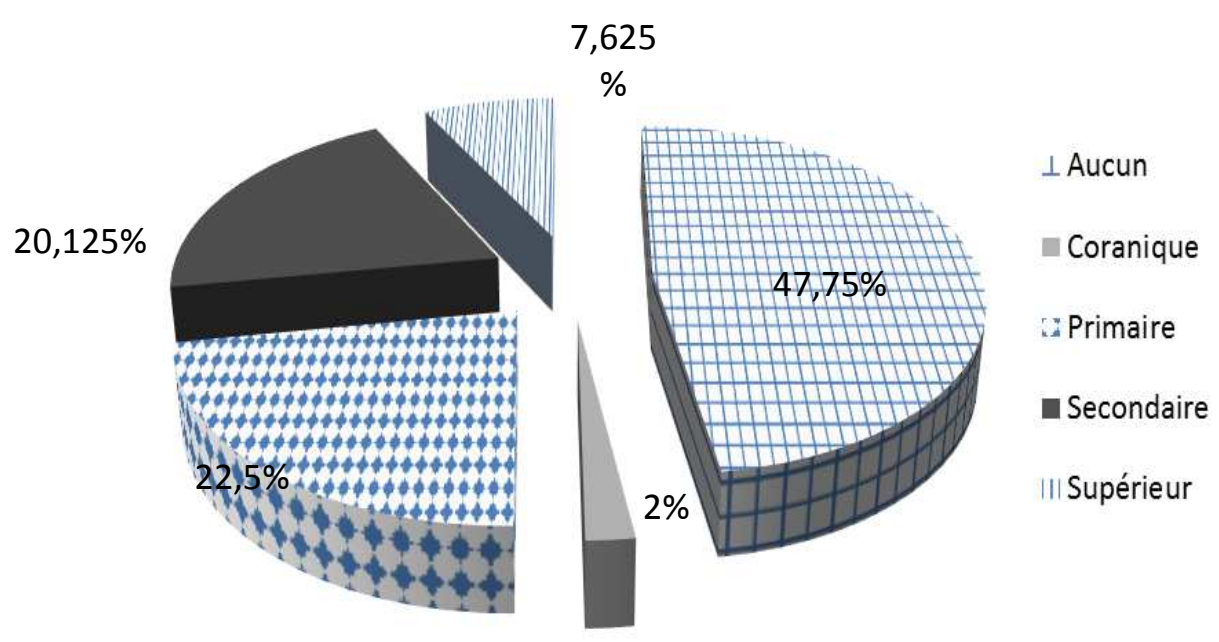

Figure 8 : Diagramme montrant le niveau d'instruction des ménages interrogés.

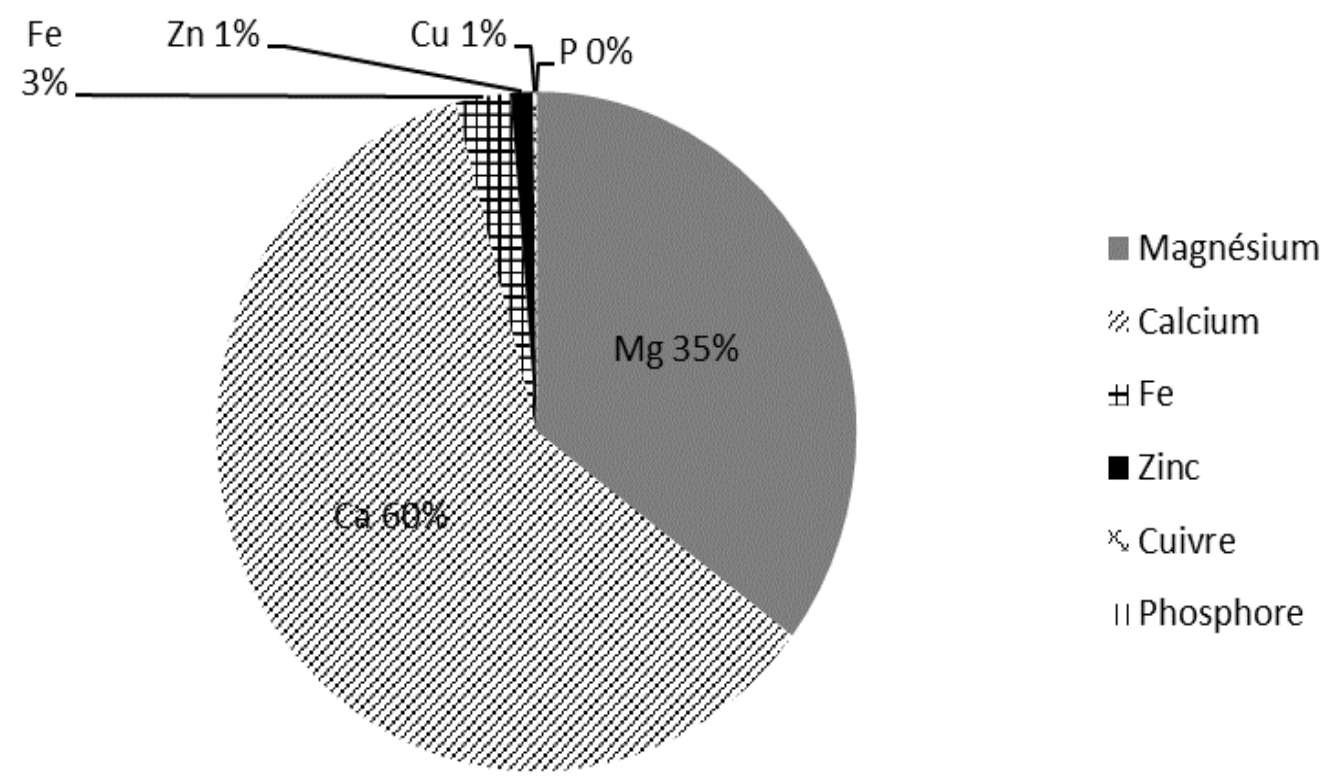

Figure 9 : Diagramme des éléments minéraux contenus dans Hydrocotyle bonariensis. $\mathrm{Ca}=$ Calcium; $\mathrm{Mg}=$ Magnésium; $\mathrm{Fe}=\mathrm{Fer} ; \mathrm{Cu}=$ Cuivre; $\mathrm{Zn}=\mathrm{Zinc} ; \mathrm{P}=$ Phosphore. 
E. D. MONYN et al. / Int. J. Biol. Chem. Sci. 10(5): 2046-2061, 2016

Tableau 1 : Caractéristiques sociodémographiques des personnes interrogées.

\begin{tabular}{|c|c|c|c|c|c|c|c|c|}
\hline \multirow{2}{*}{ Paramètres } & \multicolumn{6}{|c|}{ Zone d'étude } & \multirow{2}{*}{ Total } & \multirow{2}{*}{ Pourcentage } \\
\hline & Abobo & Anyama & Yopougon & Port-Bouët & Bingerville & Cocody & & \\
\hline \multicolumn{9}{|c|}{ Niveau d'étude } \\
\hline Aucun & 87 & 72 & 61 & 58 & 83 & 21 & 382 & 47,75 \\
\hline Primaire & 31 & 32 & 37 & 38 & 38 & 4 & 180 & 22,50 \\
\hline Secondaire & 18 & 25 & 37 & 44 & 23 & 14 & 161 & 20,13 \\
\hline Supérieur & 5 & 16 & 15 & 9 & 6 & 10 & 61 & 7,63 \\
\hline Coranique & 9 & 5 & 0 & 1 & 0 & 1 & 16 & 2,00 \\
\hline \multicolumn{9}{|c|}{ Groupe ethnique } \\
\hline Akan Sud & 14 & 16 & 25 & 21 & 33 & 5 & 114 & 14,25 \\
\hline Akan Est & 14 & 19 & 15 & 21 & 20 & 9 & 98 & 12,25 \\
\hline Akan Centre & 24 & 19 & 30 & 29 & 25 & 10 & 137 & 17,13 \\
\hline Mandé Sud & 8 & 6 & 26 & 10 & 15 & 3 & 68 & 8,50 \\
\hline Mandé Nord & 29 & 38 & 9 & 10 & 9 & 5 & 100 & 12,50 \\
\hline Krou & 22 & 21 & 27 & 35 & 23 & 11 & 139 & 17,38 \\
\hline Gour & 12 & 16 & 7 & 5 & 3 & 2 & 45 & 5,63 \\
\hline Autres & 27 & 15 & 11 & 19 & 22 & 5 & 99 & 12,38 \\
\hline \multicolumn{9}{|l|}{ Classe d'âge } \\
\hline$[18-30]$ & 18 & 11 & 2 & 1 & 2 & 1 & 35 & 4,38 \\
\hline [31-45] & 62 & 64 & 68 & 71 & 64 & 22 & 351 & 43,88 \\
\hline$>46$ & 70 & 75 & 80 & 78 & 84 & 27 & 414 & 51,75 \\
\hline \multicolumn{9}{|c|}{ Situation sociale } \\
\hline Commerçant & 74 & 74 & 69 & 21 & 52 & 60 & 350 & 43,76 \\
\hline
\end{tabular}


E. D. MONYN et al. / Int. J. Biol. Chem. Sci. 10(5): 2046-2061, 2016

\begin{tabular}{lrrrrrrrr} 
Etudiant & 3 & 2 & 2 & 1 & 0 & 4 & 12 & 1,50 \\
Fonctionnaire & 14 & 15 & 16 & 8 & 21 & 20 & 94 & 11,75 \\
Ménagère & 58 & 58 & 58 & 20 & 75 & 64 & 333 & 41,62 \\
Sans emploi & 1 & 1 & 5 & & 2 & 2 & 11 & 1,38 \\
\hline Sexe & & & & & & & & \\
Féminin & 108 & 100 & 127 & 36 & 119 & 107 & 597 & 74,62 \\
Masculin & 42 & 50 & 23 & 14 & 31 & 43 & 203 & 25,38 \\
\hline
\end{tabular}

Tableau 2 : Indices de consensus d'utilisation médicinale des feuilles de Hydrocotyle bonariensis

\begin{tabular}{lc}
\hline Pathologies & Indice de consensus \\
\hline Diabète & 0,01 \\
Hypertension & 0,12 \\
Maladies immunitaires & 0,27 \\
Croissance & 0,04 \\
Maladies nutritionnelles & 0,01 \\
Maladies de la peau & 0,006 \\
Maladies dégénératives (liées à l'âge) & 0,07 \\
Maladies du système nerveux & 0,08 \\
Maladies rhumatismales & 0,04 \\
Maladies liées à la reproduction & 0,08 \\
Maladies du système circulatoire & 0,03 \\
Maladies du système digestif & 0,17 \\
Maladies infectieuses & 0,02 \\
Maladies du système respiratoire & 0,06 \\
\hline
\end{tabular}


Tableau 3 : Indices de consensus d'utilisation alimentaire des feuilles de Hydrocotyle bonariensis.

\begin{tabular}{lc}
\hline Modes de préparation & Indice de consensus \\
\hline Crudité & 0,88 \\
Tisane & 0,006 \\
Légumes & 0,26 \\
\hline
\end{tabular}

Tableau 4 : Teneurs en minéraux des feuilles de Hydrocotyle bonariensis.

\begin{tabular}{lc}
\hline Minéraux & $\begin{array}{c}\text { Quantité (mg/100 g de matière } \\
\text { sèche) }\end{array}$ \\
\hline Calcium & 340,84 \\
Magnésium & 198,23 \\
Fe & 15,62 \\
Zinc & 6,36 \\
Cuivre & 0,97 \\
Phosphore & 0 \\
\hline
\end{tabular}

\section{DISCUSSION}

L'objectif de ce travail a été d'inventorier les usages de Hydrocotyle bonariensis dans les ménages de six communes du district d'Abidjan, puis de déterminer la composition en micronutriments des feuilles.

Les enquêtes ethnobotaniques ont montré que $64 \%$ des ménages interviewés connaissent la plante. Plus de 52\% l'utilisent soit à des fins médicinales, soit dans l'alimentation. Le niveau de connaissance et d'utilisation est statistiquement différent dans les communes. Abobo, Anyama et Cocody ont donné un niveau faible (entre 40 et 50\%) comparées à celles de Yopougon, Port-Bouët et Bingerville qui ont des forts taux (entre 70 et $90 \%$ ). La connaissance de la plante est donc liée à son utilisation mais aussi à la consommation. Par exemple, Bingerville est la commune où l'utilisation ainsi que la consommation sont très élevées $(90 \%)$. Cela pourrait s'expliquer par le fait que les populations de Bingerville en Côte d'Ivoire ont beaucoup recours aux plantes aussi bien alimentaires que médicinales dans leurs habitudes (N'Guessan et al., 2009 ; Séguéna et al., 2013).
Au niveau des groupes culturels par contre, les niveaux de connaissance sont approximativement égaux sauf pour le groupe des Mandé du Nord qui a une faible connaissance de cette plante (moins de 40\%). Cette connaissance n'est pas liée à l'utilisation et à la consommation comme dans le cas des communes. Il ressort que ce sont les Akan du centre qui consomment davantage $H$. bonariensis. L'origine culturelle pourrait donc être un facteur de choix dans l'utilisation des feuilles de cette plante dans l'alimentation. Cela corrobore les travaux de Yao et al. (2015) qui ont montré que l'utilisation des légumes feuilles dans l'alimentation des populations en zones urbaines de la Côte d'Ivoire sont liées à plusieurs facteurs, entre autres le groupe culturel auquel appartient de l'individu.

Le pourcentage de consommation des feuilles de $H$. bonariensis dans l'ensemble des zones d'enquêtes, est relativement faible (27\%). Ce résultat est en accord avec ceux de Atchibri et al. (2012) qui ont rapporté que les légumes-feuilles n'occupent pas une place de choix dans les habitudes alimentaires en Côte d'Ivoire. Toutefois à Bingerville, les feuilles de $H$. bonariensis sont très utilisées dans les 
ménages. Cela serait dû à leur disponibilité dans cette localité.

On note aussi que la consommation des feuilles de $H$. bonariensis se fait surtout sous forme de crudité et est beaucoup influencée par le niveau d'instruction et de vie. Les ménages à niveau d'instruction bas consomment plus cette plante que ceux à niveau élevé. Selon Tchiégang et Kitikil (2004), ceci tient au fait que les personnes avec un niveau faible d'instruction restent plus attachées à leurs valeurs culturelles. Tandis que celles à niveau élevé ont tendance à adopter des modes de vie occidentaux.

L'âge de l'individu pourrait être un facteur influençant le niveau de connaissance ; ce qui justifierait que les personnes ayant plus de 45 ans occupent la plus forte proportion dans cette étude. Ceci est peut-être lié aux maladies traitées par $H$. bonariensis qui affectent souvent les personnes âgées.

Le caractère bénéfice santé est également un facteur qui oriente beaucoup le choix des feuilles de Hydrocotyle bonariensis pour son utilisation dans ces ménages du district d'Abidjan. Cette plante est souvent utilisée pour soigner diverses maladies telles que l'hypertension, les maladies du système immunitaire, les maladies du système digestif ou comme tonifiant, etc. Ces usages corroborent son utilisation dans la médecine populaire américaine (Fiaschi, 2010). Plusieurs consommateurs interrogés ont déclaré que la consommation régulière des feuilles de $H$. bonariensis est dû au fait que cette plante contribue à l'amélioration de leur état de santé. La recherche des effets promoteurs de la santé suite à la consommation de $H$. bonariensis en zones urbaines concorde avec les résultats de Yao et al. (2015). Selon ces auteurs, les populations urbaines introduisent les légumes feuilles comme complément de leur régime alimentaire en raison de leur bénéfice pour la santé.

L'étude de la composition en oligoéléments des feuilles de $H$. bonariensis a montré qu'elles contiennent du calcium, du magnésium, du fer, du zinc, et du cuivre. La présence de ces minéraux est étroitement liée aux utilisations médicinales indiquées par les personnes interrogées. Chacun de ces micronutriments joue un rôle important dans le métabolisme de l'organisme. Le calcium a un effet sur la santé des os en termes de protection (Park et Weaver, 2012); le magnésium peut faire baisser la tension artérielle, le taux du cholestérol et est impliqué dans les systèmes de défenses naturelles contre les agressions de toute sorte; il est très important pour la femme enceinte (Sidor et al., 2016). Le fer a un rôle essentiel dans le métabolisme des globules rouges (hémoglobine), dans la fonction musculaire. L'anémie par carence en fer est la plus fréquente chez les nourrissons et les enfants en Afrique subsaharienne (Paganini et al., 2016). Le cuivre est impliqué dans la lutte contre les radicaux libres. Le zinc est l'un des plus importants éléments impliqués dans le métabolisme chez l'Homme. C'est un minéral antioxydant, stabilisateur des membranes lipidiques, et qui joue un rôle dans les fonctions reproductrices et dans le maintien du matériel génétique (Rahman et al., 2016). Toutes ces propriétés biologiques et physiologiques montrent l'intérêt des populations pour $H$. bonariensis dans la lutte contre les carences en micronutriments et autres maladies.

\section{Conclusion}

Les enquêtes ethnobotaniques menées dans le district d'Abidjan révèlent que les feuilles d'Hydrocotyle bonariensis entrent dans les habitudes alimentaires et médicinales des ménages. Ces résultats montrent que les espèces spontanées continuent de contribuer à la nutrition et à la santé en zones urbaines en Côte d'Ivoire, malgré souvent l'adoption de comportements nouveaux. Par ailleurs, cette plante est riche en micronutriments indispensables au bon fonctionnement de l'organisme.

$\mathrm{Au}$ vu de ces résultats, cette ressource naturelle serait pour les populations d'un 
intérêt non négligeable afin de venir à bout de nombreuses pathologies. Il serait nécessaire de promouvoir une large consommation de cette plante dans les ménages en raison de son bénéfice santé certain.

\section{REMERCIEMENTS}

Nous remercions l'Institut Polytechnique de Yamoussoukro pour l'analyse de la composition chimique de la plante étudiée. Nos remerciements vont également à l'endroit des populations du district d'Abidjan pour avoir autorisé l'accès à leurs domiciles et leur accueil chaleureux.

\section{CONFLIT D'INTERETS} d'intérêt

Les auteurs ne déclarent aucun conflit

\section{CONTRIBUTION DES AUTEURS}

EDM a rédigé le questionnaire, mené les enquêtes auprès des ménages, pris part à la rédaction et a approuvé la version finale du manuscrit. $\mathrm{AB}$ a procédé à l'identification de l'espèce végétale, pris part à la rédaction et la correction et approuvé la version finale du manuscrit. FHTB a co-supervisé les enquêtes sur le terrain, pris part à la rédaction et la correction et approuvé la version finale du manuscrit. KY s'est occupé des analyses statistiques. Il a approuvé la version finale du manuscrit. MWK a développé le concept de l'étude, guidé la confection du questionnaire, supervisé les enquêtes sur le terrain, pris part à la rédaction et la correction et a approuvé la version finale du manuscrit.

\section{REFERENCES}

Acho CF, Zoue LT, Akpa EE, Yapo VG, Niamke SL. 2014. Leafy vegetables consumed in Southern Côte d'Ivoire: a source of high value nutrients. J. Anim. Plant Sci., 20(3): 3159-3170.

Adeniyi SA, Orjiekwe CL, Ehiagbonare JE, ARIMAH BD. 2012. Evaluation of chemical composition of the leaves of Ocimum gratissimum and Vernonia amygdalina. Int. J. Biol. Chem. Sci., 6(3): 1316-1323.

Adjatin A, Dansi A, Badoussi E, Sanoussi AF, Gbaguidi F, Azokpota P, Vodouhe R, Akoègninou A, Akpagana K, Sanni A. 2013. Proximate, mineral and vitamin C composition of vegetable Gbolo [Crassocephalum rubens (Juss. Ex Jacq.) S. Moore and $C$. crepidioides (Benth.) S. Moore] in Benin. Int. J. Biol. Chem. Sci., 7(1): 319-331.

Ambé GA. 2001. Contribution à l'inventaire des plantes sauvages utiles des savanes guinéennes de la Côte d'Ivoire : cas du village de Kênêgbê. Certificat d'Études Approfondies Université de Gembloux, Gembloux, 76 p.

Andrea J. 2010. Instituto de Pesquisas Jardim Botânico do Rio de Janeiro. http://www.jbrj.gov.br/publica/livros_p df/plantas_fungos_vol1.pdf. (Consulté le 2 septembre 2016).

AOAC. 1990. Official Methods of Analysis. Association of official Analytical chemists, food composition, Official Analytical Chemists, additives natural contaminant (Vol 2, 15e édn). Adrich RC (ed). Association des Officiels Chimistes Analytiques, Inc USA : Washington.

Atchibri ALO-A, Soro LC, Kouamé C, Agbo EA, Kouadio KKA. 2012. Valeur nutritionnelle des légumes feuilles consommés en Côte d'Ivoire. Int. J. Biol. Chem. Sci., 6(1): 128-135.

Diarra N, Togola A, Denou A, Willcox M, Daou C, Diallo D. 2016. Etude ethnobotanique des plantes alimentaires utilisées en période de soudure dans les régions Sud du Mali. Int. J. Biol. Chem. Sci., 10(1): 184-197.

Dozon J-P. 1997. L'étranger et l'allochtone en Côte-d'Ivoire. In Le Modèle Ivoirien en Questions: Crises, Ajustements, Recompositions Contamin Bernard, Memel-Fotê H (eds). Karthala; Orstom : Paris ; 779-798. 
FAO (Programme alimentaire mondial). 2016. Lutte contre la faim. https://fr.wfp.org/faim/faq consulté le 1 septembre 2016.

Fiaschi P. 2010. Liste des espèces de la flore du brésil, Araliaceae. Jardin botanique de Rio de Janeiro.

Guarrera PM, Savo V. 2016. Wild food plants used in traditional vegetable mixtures in Italy. J. Ethnopharmacol., 185: 20234.

Hailu AA, Addis G. 2016. The Content and Bioavailability of Mineral Nutrients of Selected Wild and Traditional Edible Plants as Affected by Household Preparation Methods Practiced by Local Community in Benishangul Gumuz Regional State, Ethiopia. Inter. J. Food Sci., 7 p.

IITA. 1981. International Institute of Tropical Agriculture. Automated and semiautomated methods for soil and plant analysis. Manual series IITA: Ibadan.

Ilumbe GB, Van Damme P, Lukoki FL, Joiris V, Visser M, Lejoly J. 2014. Contribution à l'étude des plantes médicinales dans le traitement des hémorroïdes par les pygmées Twa et leur voisin Oto de Bikoro, en RDC. Congo Sci., 2: 46- 54.

INS. 2015. Recensement général de la population et des habitations 2014, données socio- démographiques des localités. Institut National de Statistiques : Abidjan.

Kouamé NMT, Soro K, Mangara A, Diarrassouba N, Coulibaly AV, Boraud NKM. 2015. Étude physico-chimique de sept (7) plantes spontanées alimentaires du centre-ouest de la Côte d'Ivoire. J. Appl. Biosci., 90: 8450 8463.

Kouassi JB, Massara C-C, Sess DE, Tiahou G, Djohan FY. 2013. Détermination des teneurs en magnésium, potassium, manganèse et sodium de gombo. $J$ Appl Biosci., 67: 5219-5227.
N'Guessan K, Kadja B, Zirihi GN, Traore D, Aké-Assi L. 2009. Screening phytochimique de quelques plantes médicinales ivoiriennes utilisées en pays Krobou (Agboville, Côte d'Ivoire). Sci. Nat., 1(6): 1-15.

Paganini D, Uyoga MA, Zimmermann MB. 2016. Iron Fortification of Foods for Infants and Children in Low-Income Countries: Effects on the Gut Microbiome, Gut Inflammation, and Diarrhea. Nutrients, 8(8): 494.

Park CY, Weaver CM. 2012. Review Vitamin D Interactions with Soy Isoflavones on Bone after Menopause: A Review. Nutrients., 4: 1610-1621.

Petropoulos SA, Levizou E, Ntatsi G, Fernandes $\hat{A}$, Petrotos K, Akoumianakis K, Barros L, Ferreira IC. 2017. Salinity effect on nutritional value, chemical composition and bioactive compounds content of Cichorium spinosum L. Food Chem., 214: 129-36.

Rahman S, Ahmed T, Rahman AS, Alam N, Ahmed AMS, Ireen S, Chowdhury IA, Chowdhury FP, Rahman SMM. 2016. Status of zinc nutrition in Bangladesh: the underlying associations. J. Nutr. Sci., 5(e25): 1-9.

Sanclemente T, Marques-Lopes I, FajóPascual M, Cofán M, Jarauta E, Ros E, Puzo J, García- Otín AL. 2011. Naturally-occurring phytosterols in the usual diet influence cholesterol metabolism in healthy subjects. Nutr. Metab. Cardiovasc. Dis., 22(10): 84955.

Séguéna F, Soro K, Soro D, N'Guessan K. 2013. Savoir -faire des populations locales des taxons du jardin Botanique de Bingerville, Côte d'Ivoire. J. Appl. Biosci., 68: 5374-5393.

Sidor P, Głąbska D, Włodarek D. 2016. Analysis of the dietary factors contributing to the future osteoporosis risk in young Polish women. Rocz. Panstw. Zakl. Hig., 67(3): 279-85. 
Soro LC, Atchibri ALO-A, Kouadio KKA, Kouamé C. 2012. Evaluation de la composition nutritionnelle des légumes feuilles. J. Appl. Biosci., 51: 35673573.

Sprengel KPJ. 1820. Hydrocotyle bonariensis. In Systema Vegetabilium (6th Edition) C von Linné (ed). Stuttgart, Allemagne.

Tchiegang C, Kitikil A. 2004. Données ethnonutritionnelles et caractéristiques physico- chimiques des légumes feuilles consommés dans la savane de l'Adamaoua (Cameroun) Tropicultura., 22(1): 11-18.

Vennetier P. 1983. Atlas de la Côte d'Ivoire ( $2^{\mathrm{e}}$ éd.). Les atlas Jeune Afrique : Paris
Vessereau A., 1992. Méthodes Statistiques en Biologie et en Agronomie. TEC \& DOC Lavoisier : Paris.

Volpe MG, Nazzaro M, Di Stasio M, Siano F, Coppola R, De Marco A. 2015. Content of micronutrients, mineral and trace elements in some Mediterranean spontaneous edible herbs. Chem Cent J., 9: 57.

Yao K, Koné MW, Kamanzi K. 2015. Contribution des Légumes Feuilles à la Nutrition des Populations en Zones Urbaines de la Côte D'ivoire. Eur. J. Sci. Res., 130(4): 338-351. 